\title{
MULTIPLICITY OF SOLUTIONS FOR ELLIPTIC PROBLEMS WITH CRITICAL EXPONENT OR WITH A NONSYMMETRIC TERM
}

\section{J. GARCIA AZORERO AND I. PERAL ALONSO}

ABstract. We study the existence of solutions for the following nonlinear degenerate elliptic problems in a bounded domain $\Omega \subset \mathbf{R}^{N}$

$$
-\operatorname{div}\left(|\nabla u|^{p-2} \nabla u\right)=|u|^{p^{*}-2} u+\lambda|u|^{q-2} u, \quad \lambda>0,
$$

where $p^{*}$ is the critical Sobolev exponent, and $\left.u\right|_{\delta \Omega} \equiv 0$. By using critical point methods we obtain the existence of solutions in the following cases:

If $p<q<p^{*}$, there exists $\lambda_{0}>0$ such that for all $\lambda>\lambda_{0}$ there exists a nontrivial solution.

If $\max \left(p, p^{*}-p /(p-1)\right)<q<p^{*}$, there exists nontrivial solution for all $\lambda>0$.

If $1<q<p$ there exists $\lambda_{1}$ such that, for $0<\lambda<\lambda_{1}$, there exist infinitely many solutions.

Finally, we obtain a multiplicity result in a noncritical problem when the associated functional is not symmetric.

\section{INTRODUCTION}

In this work we will consider the following model problem:

$$
\begin{gathered}
-\operatorname{div}\left(|\nabla u|^{p-2} \nabla u\right)=|u|^{p^{*}-2} u+\lambda|u|^{q-2} u, \\
\left.u\right|_{\delta \Omega}=0,
\end{gathered}
$$

where $\lambda>0, \Omega$ is a bounded domain in $\mathbf{R}^{N}$ with boundary $\delta \Omega$, and assume that

(i) $1<p<N$,

(ii) $p^{*}=p N /(N-p)$,

(iii) $1<q<p^{*}$.

Observe that $p^{*}$ is the critical exponent in the Sobolev inclusion theorem. The nonlinear differential operator is called $p$-Laplacian, $\Delta_{p}$. We look for nontrivial

Received by the editors February 16, 1989.

1980 Mathematics Subject Classification (1985 Revision). Primary 35J20, 35J25, 35J60; Secondary $35 \mathrm{~J} 65,35 \mathrm{~J} 70$.

Both authors were partially supported by Dirección General de Investigación Cientifica y Técnica (MEC, España) Project PB86-0112-C02-00. 
solutions of (1.1), and this question is reduced to show the existence of critical points for the functional

$$
F(u)=\frac{1}{p} \int_{\Omega}|\nabla u|^{p}-\frac{\lambda}{q} \int_{\Omega}|u|^{q}-\frac{1}{p^{*}} \int_{\Omega}|u|^{p^{*}} .
$$

Under hypothesis (1.2), $F(u)$ is defined on the Sobolev space $W_{0}^{1, p}(\Omega)$.

By using the so-called generalized Pohozaev identity, it is possible to prove that, if the domain $\Omega$ is starshaped, then (1.1) cannot have any nontrivial solution in $W_{0}^{1, p}(\Omega)$ if $\lambda \leq 0$ (see [P-S], and also [O, G-V, and E]); therefore, we are reduced to consider positive $\lambda$.

For $p=q$ the problem is studied in [G-P.1] where the existence of positive solution for the dimensions $N$ such that $p^{2} \leq N$ is obtained, if $0<\lambda<\lambda_{1}, \lambda_{1}$ being the first eigenvalue for the $p$-Laplacian $\left(\lambda_{1}\right.$ is isolated and simple, as it is obtained in [Ba]; see also [Bha and A]). The main difficulty in solving problem (1.1) is the lack of compactness in the inclusion of $W_{0}^{1, p}(\Omega)$ in $L^{p^{*}}$, because in general the Palais-Smale condition is not satisfied.

In the case $p=2$, the problem has been solved by Brézis-Nirenberg [B-N]. As in [B-N], we obtain a local Palais-Smale condition for the case $p \neq 2$ which is sufficient. This question is handled in $\S 2$ by the concentration-compactness principle of $\mathrm{P}$. L. Lions (see [L1 and L2]).

In $\S 3$ we analyze the case $p<q<p^{*}$ and achieve some new results with respect to those obtained in [G-P.1].

The case $1<q<p$ is managed in $\S 4$ by classical critical point theory. See [B-F and G-P.1] for related methods in the subcritical case.

Obviously, more general terms can be handled if their behaviour at 0 and at infinity is the same.

Finally, in $\S 5$, we solve some nonsymmetric problems. Following the ideas in [R1] we also obtain multiplicity results in this case.

For the regularity of the solutions, see [T and DiB].

\section{The Palais-Smale condition}

A sequence $\left\{u_{j}\right\} \subset W_{0}^{1, p}(\Omega)$ is called a Palais-Smale sequence for $F$, defined by (1.3), if

$$
\begin{gathered}
F\left(u_{j}\right) \rightarrow c, \\
F^{\prime}\left(u_{j}\right) \rightarrow 0 \quad \text { in } W^{-1, p^{\prime}}(\Omega), \text { where } 1 / p+1 / p^{\prime}=1 .
\end{gathered}
$$

If $(2.1)$ implies the existence of a subsequence $\left\{u_{j_{k}}\right\} \subset\left\{u_{j}\right\}$ which converges in $W_{0}^{1, p}(\Omega)$, we say that $F$ verifies the Palais-Smale condition.

If this strongly convergent subsequence exists only for some $c$ values, we say that $F$ verifies a local Palais-Smale condition.

In our case, the main difficulty is the lack of compactness in the inclusion of $W_{0}^{1, p}(\Omega)$ in $L^{p^{*}}$. Then, we prove a local Palais-Smale condition, which is sufficient although with some restrictions. 
The technical results which we must use are based on a measure representation lemma, used by P. L. Lions in the proof of the concentration-compactness principle (see [L1 and L2]).

Let $\left\{u_{j}\right\}$ be a bounded sequence in $W_{0}^{1, p}(\Omega)$. Then, there is a subsequence, such that $u_{j} \rightarrow u$, weakly in $W_{0}^{1, p}(\Omega)$, and

$$
\begin{aligned}
& \left|\nabla u_{j}\right|^{p}-d \mu \\
& \left|u_{j}\right|^{p^{*}}-d \nu
\end{aligned} \quad \text { weakly-*}{ }^{*} \text { in the sense of measures. }
$$

If we take $\varphi \in C_{0}^{\infty}\left(\mathbf{R}^{N}\right)$, by some calculations with the Sobolev inequality we conclude that

$$
\left(\int_{\Omega}|\varphi|^{p^{*}} d \nu\right)^{1 / p^{*}} S^{1 / p} \leq\left(\int_{\Omega}|\varphi|^{p} d \mu\right)^{1 / p}+\left(\int_{\Omega}|\nabla \varphi|^{p}|u|^{p} d x\right)^{1 / p}
$$

where

$$
S=\inf \left\{\|u\|_{W_{0}^{1, p}(\Omega)}: u \in W_{0}^{1, p}(\Omega),\|u\|_{p^{*}}=1\right\}
$$

is the best constant in the Sobolev inclusion.

If, in (2.2), we suppose $u \equiv 0$, then we have a reverse Hölder inequality for two differents measures. In this situation, we have the following representation of the measures (see P. L. Lions [L1 and L2]):

Lemma 2.1. Let $\mu, \nu$ be two nonnegative and bounded measures on $\bar{\Omega}$, such that for $1 \leq p<r<\infty$ there exists some constant $C>0$ such that

$$
\left(\int_{\Omega}|\varphi|^{r} d \nu\right)^{1 / r} \leq C\left(\int_{\Omega}|\varphi|^{p} d \mu\right)^{1 / p} \quad \forall \varphi \in C_{0}^{\infty}\left(\mathbf{R}^{N}\right) .
$$

Then, there exist $\left\{x_{j}\right\}_{j \in J} \subset \bar{\Omega}$ and $\left\{\nu_{j}\right\}_{j \in J} \subset(0, \infty)$, where $J$ is at most countable, such that

$$
\nu=\sum_{j \in J} \nu_{j} \delta_{x_{j}}, \quad \mu \geq C^{-p} \sum_{j \in J} \nu_{j}^{p / r} \delta_{x_{j}},
$$

where $\delta_{x_{j}}$ is the Dirac mass at $x_{j}$.

If we apply this lemma to $v_{j}=u_{j}-u$, we obtain the following result, due to P. L. Lions (see [L1 and L2]):

Lemma 2.2. Let $\left\{u_{j}\right\}$ be a weakly convergent sequence in $W_{0}^{1, p}(\Omega)$ with weak limit $u$, and such that

(i) $\left|\nabla u_{j}\right|^{p}$ converges in the weak-* sense of measures to a measure $\mu$,

(ii) $\left|u_{j}\right|^{p^{*}}$ converges in the weak-* sense of measures to a measure $\nu$.

Then, for some at most countable index set $J$ we have

$$
\begin{array}{ll}
\text { (1) } \nu=|u|^{p^{*}}+\sum_{j \in J} \nu_{j} \delta_{x_{j}}, & \nu_{j}>0, \\
\text { (2) } \mu \geq|\nabla u|^{p}+\sum_{j \in J} \mu_{j} \delta_{x_{j}}, & \mu_{j}>0, \\
\text { (3) } \nu_{j}^{p / p^{*}} \leq \mu_{j} / S,
\end{array}
$$

where $x_{j} \in \bar{\Omega}$. 
The relations (2.3) with the hypothesis that the constant $c$ in (2.1) is small enough allow us to prove that the singular part of the measures must be 0 , and we have a local Palais-Smale condition.

Lemma 2.3. Let $\left\{v_{j}\right\} \subset W_{0}^{1, p}(\Omega)$ be a Palais-Smale sequence for $F$, defined by (1.3), that is,

$$
\begin{gathered}
F\left(v_{j}\right) \rightarrow C, \\
F^{\prime}\left(v_{j}\right) \rightarrow 0 \quad \text { in } W^{-1, p^{\prime}}(\Omega), \quad 1 / p+1 / p^{\prime}=1 .
\end{gathered}
$$

Then, we have

(a) If $p<q<p^{*}$, and $C<S^{N / p} / N$, there exists a subsequence $\left\{v_{j_{k}}\right\} \subset\left\{v_{j}\right\}$, strongly convergent in $W_{0}^{1, p}(\Omega)$.

(b) If $1<q<p$, and $C<S^{N / p} / N-K \lambda^{\beta}$, where $\beta=p^{*} /\left(p^{*}-q\right)$ and $K$ depends on $p, q, N$ and $\Omega$, then there exists a subsequence $\left\{v_{j_{k}}\right\} \subset\left\{v_{j}\right\}$, strongly convergent in $W_{0}^{1, p}(\Omega)$.

Proof. In both cases, by (2.4) and (2.5), it is easy to prove that the sequence $\left\{v_{j}\right\}$ is bounded in $W_{0}^{1, p}(\Omega)$. Then, if we take the appropriate subsequence, we can assume in both cases (by Lemma 2.2)

$$
\begin{gathered}
v_{j} \rightarrow v \text { weakly in } W_{0}^{1, p}(\Omega), \\
v_{j} \rightarrow v \text { in } L^{r}, \quad 1<r<p^{*}, \text { and a.e. }, \\
\left|\nabla v_{j}\right|^{p}-d \mu \geq|\nabla v|^{p}+\sum_{k \in J} \mu_{k} \delta_{x_{k}}, \\
\left|v_{j}\right|^{p^{*}}-d \nu=|v|^{p^{*}}+\sum_{k \in J} \nu_{k} \delta_{x_{k}} .
\end{gathered}
$$

Take $x_{k} \in \bar{\Omega}$ in the support of the singular part of $d \mu, d \nu$. We consider $\varphi \in C_{0}^{\infty}\left(\mathbf{R}^{N}\right)$, such that

$$
\varphi \equiv 1 \text { on } B\left(x_{k}, \varepsilon\right), \quad \varphi \equiv 0 \text { on } B\left(x_{k}, 2 \varepsilon\right)^{c}, \quad|\nabla \varphi| \leq 2 / \varepsilon .
$$

It is clear that the sequence $\left\{\varphi v_{j}\right\}$ is bounded in $W_{0}^{1, p}(\Omega)$; then, by using hypothesis $(2.5), \lim \left\langle F^{\prime}\left(v_{j}\right), \varphi v_{j}\right\rangle=0(\langle$,$\rangle is the duality product )$, and

$$
\int \varphi d \nu+\lambda \int|v|^{q} \varphi d x-\int \varphi d \mu=\lim _{j} \int\left|\nabla v_{j}\right|^{p-2} v_{j}\left(\nabla v_{j}, \nabla \varphi\right) d x
$$

$\left((\cdot, \cdot)\right.$ is the product in $\left.\mathbf{R}^{N}\right)$. By $(2.6),(2.7)$, and the Hölder inequality, we obtain

$$
0 \leq\left.\lim _{j}\left|\int\right| \nabla v_{j}\right|^{p-2} v_{j}\left(\nabla v_{j}, \nabla \varphi\right) d x \mid \leq C\left(\int_{B\left(x_{k}, 2 \varepsilon\right)}|v|^{p^{*}}\right)^{1 / p^{*}} \underset{\varepsilon \rightarrow 0}{\longrightarrow} 0 .
$$


Then,

$$
0=\lim _{\varepsilon \rightarrow 0}\left\{\int \varphi d \nu+\lambda \int|v|^{q} \varphi d x-\int \varphi d \mu\right\}=\nu_{k}-\mu_{k} .
$$

By Lemma 2.2, $\mu_{k} \geq S \nu_{k}^{p / p^{*}}$, i.e. $\nu_{k} \geq S \nu_{k}^{p / p^{*}}$. That is, $\nu_{k}=0$, or

$$
\nu_{k} \geq S^{N / p} \text {. }
$$

(In particular, there are, at most, a finite number of singularities, because $d \nu$ is a bounded measure.) We will prove that $(2.8)$ is not possible.

Let us assume that there exists a $k_{0}$ with $\nu_{k_{0}} \neq 0$ i.e. $\nu_{k_{0}} \geq S^{N / p}$. By (2.4) and (2.6),

$$
C=\lim _{j} F\left(v_{j}\right) \geq F(v)+\left(\frac{1}{p}-\frac{1}{p^{*}}\right) \sum \nu_{k} \geq F(v)+\frac{1}{N} S^{N / p} .
$$

But, by hypothesis, $C<S^{N / p} / N$; then, $F(v)<0$. In particular, $v \not \equiv 0$, and

$$
0<\frac{1}{p} \int|\nabla v|^{p}<\frac{1}{p^{*}} \int|v|^{p^{*}}+\frac{\lambda}{q} \int|v|^{q} .
$$

That is,

$$
\begin{aligned}
C & =\lim _{j} F\left(v_{j}\right)=\lim _{j}\left\{F\left(v_{j}\right)-1 / p\left\langle F^{\prime}\left(v_{j}\right), v_{j}\right\rangle\right\} \\
& \geq \frac{1}{N} \int|v|^{p^{*}}+\frac{1}{N} S^{N / p}+\lambda\left(\frac{1}{p}-\frac{1}{q}\right) \int|v|^{q} .
\end{aligned}
$$

Now we distinguish two cases:

(a) If $p<q<p^{*}$, then $C>S^{N / p} / N$, and this inequality contradicts the hypothesis for this case. Then, $\nu_{k}=0 \forall k$, and $\lim _{j} \int\left|v_{j}\right|^{p^{*}}=\int|v|^{p^{*}}$. By using (2.6), we conclude that $v_{j} \rightarrow v$ in $L^{p^{*}}$, and, finally, because of the continuity of $\Delta_{p}^{-1}, v_{j} \rightarrow v$ in $W_{0}^{1, p}(\Omega)$.

(b) If $1<q<p$, applying the Hölder inequality at (2.8), we have

$$
C \geq \frac{1}{N} S^{N / p}+\frac{1}{N} \int|v|^{p^{*}}-\lambda\left(\frac{1}{q}-\frac{1}{p}\right)|\Omega|^{\left(p^{*}-q\right) / p^{*}}\left(\int|v|^{p^{*}}\right)^{q / p^{*}} .
$$

Let $f(x)=c_{1} x^{p^{*}}-\lambda c_{2} x^{q}$. This function attains its absolute minimum (for $x>0)$ at the point $x_{0}=\left(\lambda c_{2} q / p^{*} c_{1}\right)^{1 /\left(p^{*}-q\right)}$. That is,

$$
f(x) \geq f\left(x_{0}\right)=-K \lambda^{p^{*} /\left(p^{*}-q\right)} \text {. }
$$

But this result contradicts the hypothesis; then, $\nu_{k}=0 \forall k$, and we conclude.

Remark 2.4. It is well known that it is impossible to improve this local PalaisSmale condition in case (a); we can construct a Palais-Smale sequence with $C=S^{N / p} / N$, without any convergent subsequence (see [B]).

In case (b) it is also possible to exhibit a counterexample; we construct this counterexample at the end of $\S 4$. 


\section{THE CASE $p<q<p^{*}$}

In $\S 2$, we have proved that below the level $S^{N / p} / N$, the functional $F$ verifies a local Palais-Smale condition. In this section we will use the Mountain Pass Lemma to prove the existence of a solution for problem (1.1).

We will use the following general version of the Mountain Pass Lemma (see [A-E] for the proof).

Lemma 3.1. Let $F$ be a functional on a Banach space $X, F \in C^{1}(X, \mathbf{R})$. Let us assume that there exists $r, R>0$, such that

(i) $F(u)>r, \forall u \in X$ with $\|u\|=R$,

(ii) $F(0)=0$, and $F\left(w_{0}\right)<r$ for some $w_{0} \in X$, with $\left\|w_{0}\right\|>R$.

Let us define $\mathbf{C}=\left\{g \in C([0,1] ; X): g(0)=0, g(1)=w_{0}\right\}$, and

$$
c=\inf _{g \in \mathbf{C}} \max _{t \in[0,1]} F(g(t)) \text {. }
$$

Then, there exists a sequence $\left\{u_{j}\right\} \subset X$, such that $F\left(u_{j}\right) \rightarrow c$, and $F^{\prime}\left(u_{j}\right) \rightarrow 0$ in $X^{*}$ (dual of $\left.X\right)$.

In our case, it is easy to see that $F$ verifies (i) and (ii).

If we can prove that

$$
c<S^{N / p} / N
$$

then Lemma 3.1 and Lemma 2.3 give the existence of the critical point of $F$.

To obtain (3.2), we choose $v_{0} \in W_{0}^{1, p}(\Omega)$, with

$$
\left\|v_{0}\right\|_{p^{*}}=1, \quad \lim _{t \rightarrow \infty} F\left(t v_{0}\right)=-\infty
$$

then, $\sup _{t \geq 0} F\left(t v_{0}\right)=F\left(t_{\lambda} v_{0}\right)$, for some $t_{\lambda}>0$. Thus $t_{\lambda}$ verifies

$$
0=t_{\lambda}^{p-1} \int\left|\nabla v_{0}\right|^{p}-t_{\lambda}^{p^{*}-1} \int\left|v_{0}\right|^{p^{*}}-\lambda t_{\lambda}^{q-1} \int\left|v_{0}\right|^{q}
$$

and we get

$$
0=t_{\lambda}^{q-1}\left(t_{\lambda}^{p-q}\left(\int\left|\nabla v_{0}\right|^{p}\right)-t_{\lambda}^{p^{*}-q}-\lambda \int\left|v_{0}\right|^{q}\right) .
$$

Observe that

$$
t_{\lambda}^{p^{*}-q}+\lambda \int\left|v_{0}\right|^{q} \underset{\lambda \rightarrow \infty}{\longrightarrow} \infty
$$

therefore, (3.4) implies $t_{\lambda} \underset{\lambda \rightarrow \infty}{\longrightarrow} 0$. By the continuity of $F$,

$$
\lim _{\lambda \rightarrow \infty}\left(\sup _{t \geq 0} F\left(t v_{0}\right)\right)=0
$$

then, there exists $\lambda_{0}$ such that $\forall \lambda \geq \lambda_{0}$,

$$
\sup _{t \geq 0} F\left(t v_{0}\right)<S^{N / p} / N
$$


If we take $w_{0}=t v_{0}$, with $t$ large enough to verify $F\left(w_{0}\right)<0$, we get

$$
c \leq \max _{t \in[0,1]} F\left(g_{0}(t)\right) \text { taking } g_{0}(t)=t w_{0} .
$$

Therefore, $c \leq \sup _{t \geq 0} F\left(t v_{0}\right)<S^{N / p} / N$, and we have proved estimate (3.2), for $\lambda$ large enough. Hence, we can apply Lemma 3.1 and Lemma 2.3, and we have the following result:

Theorem 3.2. If $p<q<p^{*}$, there exists $\lambda_{0}>0$ such that problem (1.1) has a nontrivial solution $\forall \lambda \geq \lambda_{0}$.

By choosing carefully the function $v_{0} \in W_{0}^{1, p}(\Omega)$ in (3.3), we can prove the following stronger result:

Theorem 3.3. If $\max \left(p, p^{*}-p /(p-1)\right)<q<p^{*}$, then there exists a nontrivial solution of problem (1.1), $\forall \lambda>0$.

Proof. The natural choice is to take an appropriated truncation of

$$
U_{\varepsilon}(x)=\left(\varepsilon+c\left|x-x_{0}\right|^{p /(p-1)}\right)^{(p-N) / p}
$$

because they are the functions in $W^{1, p}\left(\mathbf{R}^{N}\right)$ where the best constant in the Sobolev inclusion is attained. It is well known that they are the unique positives, except for translations and dilations (see [B, L1, L2]).

We can assume that $0 \in \Omega$, and consider $x_{0}=0$ at (3.5).

Let $\phi$ be a function $\phi \in C_{0}^{\infty}(\Omega)$, and $\phi(x) \equiv 1$ in a neighbourhood of the origin. We define $u_{\varepsilon}(x)=\phi(x) U_{\varepsilon}(x)$. For $\varepsilon \rightarrow 0$, the behaviour of $u_{\varepsilon}$ has to be like $U_{\varepsilon}$, and we can estimate the error we get when we take $u_{\varepsilon}$ instead of $U_{\varepsilon}$.

In this way, taking $v_{\varepsilon}=u_{\varepsilon} /\left\|u_{\varepsilon}\right\|_{p^{*}}$, we obtain the following estimates (see [B-N, G-P.1] for the details):

(1) Estimate for the gradient:

$$
\left\|\nabla v_{\varepsilon}\right\|_{p}^{p}=S+O\left(\varepsilon^{(N-p) / p}\right)
$$

(2) Estimate of $\left\|v_{\varepsilon}\right\|_{q}$ :

if $q>p^{*}(1-1 / p)$, then

$$
C_{1} \varepsilon^{((p-1) / p)(N-q(N-p) / p)} \leq\left\|v_{\varepsilon}\right\|_{q}^{q} \leq C_{2} \varepsilon^{((p-1) / p)(N-q(N-p) / p)} .
$$

If $q=p^{*}(1-1 / p)$, then

$$
C_{1} \varepsilon^{(N-p) q / p^{2}}|\log \varepsilon| \leq\left\|v_{\varepsilon}\right\|_{q}^{q} \leq C_{2} \varepsilon^{(N-p) q / p^{2}}|\log \varepsilon| .
$$

If $q<p^{*}(1-1 / p)$, then

$$
C_{1} \varepsilon^{(N-p) q / p^{2}} \leq\left\|v_{\varepsilon}\right\|_{q}^{q} \leq C_{2} \varepsilon^{(N-p) q / p^{2}} .
$$

Observe that, if $p<q<p^{*}$, then

$$
\left\|v_{\varepsilon}\right\|_{q}^{q} \underset{\varepsilon \rightarrow 0}{\longrightarrow} 0
$$


By using these estimates, we will show that there exists $\varepsilon>0$, small enough, such that

$$
\sup _{t \geq 0} F\left(t v_{\varepsilon}\right)<S^{N / p} / N
$$

Then, we conclude as in Theorem 3.2, by using Lemma 3.1 and Lemma 2.3.

Let us consider the functions

$$
g(t)=F\left(t v_{\varepsilon}\right)=\frac{t^{p}}{p} \int\left|\nabla v_{\varepsilon}\right|^{p}-\frac{t^{p^{*}}}{p^{*}}-\frac{\lambda t^{q}}{q} \int\left|v_{\varepsilon}\right|^{q},
$$

and

$$
\bar{g}(t)=\frac{t^{p}}{p} \int\left|\nabla v_{\varepsilon}\right|^{p}-\frac{t^{p^{*}}}{p^{*}}
$$

It is clear that $g(t) \underset{t \rightarrow \infty}{\longrightarrow}-\infty$; then, $\sup _{t \geq 0} F\left(t v_{\varepsilon}\right)$ is attained for some $t_{\varepsilon}>$ 0 , and

$$
0=g^{\prime}\left(t_{\varepsilon}\right)=t_{\varepsilon}^{p-1}\left(\int\left|\nabla v_{\varepsilon}\right|^{p}-t_{\varepsilon}^{p^{*}-p}-\lambda t_{\varepsilon}^{q-p} \int\left|v_{\varepsilon}\right|^{q}\right) .
$$

Therefore,

$$
\int\left|\nabla v_{\varepsilon}\right|^{p}=t_{\varepsilon}^{p^{*}-p}+\lambda t_{\varepsilon}^{q-p} \int\left|v_{\varepsilon}\right|^{q}>t_{\varepsilon}^{p^{*}-p}
$$

i.e.

$$
t_{\varepsilon} \leq\left(\int\left|\nabla v_{\varepsilon}\right|^{p}\right)^{1 /\left(p^{*}-p\right)}
$$

This inequality implies

$$
\int\left|\nabla v_{\varepsilon}\right|^{p} \leq t_{\varepsilon}^{p^{*}-p}+\lambda\left(\int\left|\nabla v_{\varepsilon}\right|^{p}\right)^{(q-p) /\left(p^{*}-p\right)}\left(\int\left|v_{\varepsilon}\right|^{q}\right) .
$$

Choosing $\varepsilon$ small enough, by (3.6) and (3.10),

$$
t_{\varepsilon}^{p^{*}-p} \geq S / 2
$$

That is, we have a lower bound for $t_{\varepsilon}$, independent of $\varepsilon$. Now, we estimate $g\left(t_{\varepsilon}\right)$.

The function $\bar{g}$ attains its maximum at $t=\left(\int\left|\nabla v_{\varepsilon}\right|^{p}\right)^{1 /\left(p^{*}-p\right)}$, and is increasing at the interval $\left[0,\left(\int\left|\nabla v_{\varepsilon}\right|^{p}\right)^{1 /\left(p^{*}-p\right)}\right]$. Then, by using (3.6), (3.11) and (3.13), we have

$$
\begin{aligned}
g\left(t_{\varepsilon}\right) & =\bar{g}\left(t_{\varepsilon}\right)-\frac{\lambda}{q} t_{\varepsilon}^{q} \int\left|v_{\varepsilon}\right|^{q} \\
& \leq \bar{g}\left(\left(\int\left|\nabla v_{\varepsilon}\right|^{p}\right)^{1 /\left(p^{*}-p\right)}\right)-\frac{\lambda}{q} t_{\varepsilon}^{q} \int\left|v_{\varepsilon}\right|^{q} \\
& \leq \frac{1}{N} S^{N / p}+C_{3} \varepsilon^{(N-p) / p}-\frac{\lambda}{q}\left(\frac{S}{2}\right)^{q /\left(p^{*}-p\right)} \int\left|v_{\varepsilon}\right|^{q} .
\end{aligned}
$$


Let us suppose $q>p^{*}(1-1 / p)$. Then, we have (3.7), and

$$
g\left(t_{\varepsilon}\right) \leq S^{N / p} / N+C_{3} \varepsilon^{(N-p) / p}-\lambda C_{1} \varepsilon^{\{((p-1) / p)(N-q(N-p) / p)\}} .
$$

If

$$
\frac{N-p}{p}>\frac{p-1}{p}\left(N-q \frac{(N-p)}{p}\right),
$$

that is, $q>p^{*}-p /(p-1)$, then for $\varepsilon$ small enough we get $g\left(t_{\varepsilon}\right)<S^{N / p} / N$, and we conclude.

Remark 3.4. If $N \geq p^{2}$, then $p^{*}-\frac{p}{p-1} \leq p^{*}\left(1-\frac{1}{p}\right) \leq p$, and if $p<q<p^{*}$, we have $q>p^{*}-\frac{p}{p-1}$. Then $q$ verifies the estimate (3.7), and we obtain the result of [G-P.1].

If $N<p^{2}$, then $p<p^{*}\left(1-\frac{1}{p}\right)<p^{*}-\frac{p}{p-1}$, and for $p<q \leq p^{*}-\frac{p}{p-1}$ the estimate is insufficient.

Remark 3.5. It is possible to prove the analogous result for the problem:

$$
\left\{\begin{array}{l}
-\operatorname{div}\left(|\nabla u|^{p-2} \nabla u\right)=|u|^{p^{*}-2} u+\lambda|u|^{q-2} u+f, \lambda>0 \\
\left.u\right|_{\delta \Omega}=0
\end{array}\right.
$$

if $f$ is small enough in the norm of $W^{-1, p^{\prime}}(\Omega)$. The proof is an adaptation of the above argument.

\section{THe CASE $1<q<p$}

In this section, we will construct a mini-max class of critical points, by using the classical concept and properties of the genus.

Let $X$ be a Banach space, and $\Sigma$ the class of the closed and symmetric with respect to the origin subsets of $X-\{0\}$. For $A \in \Sigma$, we define the genus $\gamma(A)$ by

$$
\gamma(A)=\min \left\{k \in \mathbf{N}: \exists \phi \in \mathbf{C}\left(A ; \mathbf{R}^{k}-\{0\}\right), \phi(x)=-\phi(-x)\right\} .
$$

If such a minimum does not exist then we define $\gamma(A)=+\infty$. The main properties of the genus are the following (see [R1 or R2] for the details):

Proposition 4.1. Let $A, B \in \Sigma$. Then

(1) If there exists $f \in \mathbf{C}(A, B)$, odd, then $\gamma(A) \leq \gamma(B)$.

(2) If $A \subset B$, then $\gamma(A) \leq \gamma(B)$.

(3) If there exists an odd homeomorphism between $A$ and $B$, then $\gamma(A)=$ $\gamma(B)$.

(4) If $S^{N-1}$ is the sphere in $\mathbf{R}^{N}$, then $\gamma\left(S^{N-1}\right)=N$.

(5) $\gamma(A \cup B) \leq \gamma(A)+\gamma(B)$.

(6) If $\gamma(B)<+\infty$, then $\gamma(\overline{A-B}) \geq \gamma(A)-\gamma(B)$.

(7) If $A$ is compact, then $\gamma(A)<+\infty$, and there exists $\delta>0$ such that $\gamma(A)=\gamma\left(N_{\delta}(A)\right)$ where $N_{\delta}(A)=\{x \in X: d(x, A) \leq \delta\}$. 
(8) If $X_{0}$ is a subspace of $X$ with codimension $K$, and $\gamma(A)<K$, then $A \cap X_{0} \neq \varnothing$.

Given the functional $F$, defined by (1.3), under the hypothesis $q<p$, by Sobolev's inequality we obtain

$$
F(u) \geq \frac{1}{p} \int|\nabla u|^{p}-\frac{1}{p^{*} S^{p^{*} / p}}\left(\int|\nabla u|^{p}\right)^{p^{*} / p}-\frac{\lambda}{q} C_{p, q}\left(\int|\nabla u|^{p}\right)^{q / p} .
$$

If we define

$$
h(x)=\frac{1}{p} x^{p}-\frac{1}{p^{*} S^{p^{*} / p}} x^{p^{*}}-\frac{\lambda}{q} C_{p, q} x^{q}
$$

then

$$
F(u) \geq h\left(\|\nabla u\|_{p}\right) .
$$

There exists $\lambda_{1}>0$ such that, if $0<\lambda \leq \lambda_{1}, h$ attains its positive maximum (see Figure 4.1).

Let us assume $0<\lambda \leq \lambda_{1}$; choosing $R_{0}$ and $R_{1}$ as in Figure 4.1 we make the following truncation of the functional $F$ :

Take $\tau: \mathbf{R}^{+} \rightarrow[0,1]$, nonincreasing and $\mathbf{C}^{\infty}$, such that

$$
\begin{array}{ll}
\tau(x)=1 & \text { if } x \leq R_{0}, \\
\tau(x)=0 & \text { if } x \geq R_{1} .
\end{array}
$$

Let $\varphi(u)=\tau\left(\|\nabla u\|_{p}\right)$. We consider the truncated functional

$$
J(u)=\frac{1}{p} \int|\nabla u|^{p}-\frac{1}{p^{*}} \int|u|^{p^{*}} \varphi(u)-\frac{\lambda}{q} \int|u|^{q} .
$$

As in (4.1), $J(u) \geq \bar{h}\left(\|\nabla u\|_{p}\right)$, with

$$
\bar{h}(x)=\frac{1}{p} x^{p}-\frac{1}{p^{*} S^{p^{*} / p}} x^{p^{*}} \tau(x)-\frac{\lambda}{q} C_{p, q} x^{q}
$$

(see Figure 4.2).

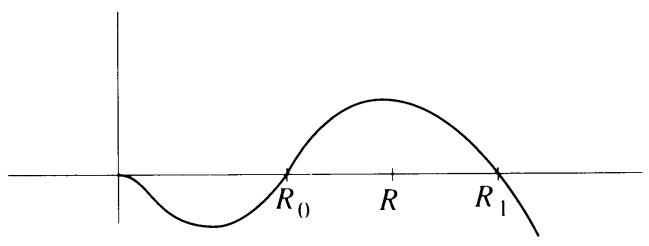

FIGURE 4.1

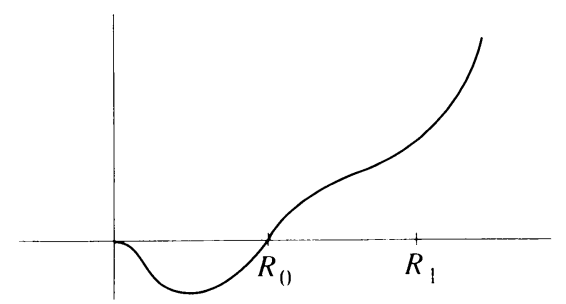

FIGURE 4.2 
Observe that for $x \leq R_{0}, \bar{h}=h$, and for $x \geq R_{1}$,

$$
\bar{h}(x)=\frac{1}{p} x^{p}-\frac{\lambda}{q} C_{p, q} x^{q} .
$$

The principal properties of $J$ defined by (4.2) are:

Lemma 4.2. (1) $J \in \mathbf{C}^{1}\left(W_{0}^{1, p}(\Omega), \mathbf{R}\right)$.

(2) If $J(u) \leq 0$, then $\|\nabla u\|_{p}<R_{0}$, and $F(v)=J(v)$ for all $v$ in a small enough neighbourhood of $u$.

(3) There exists $\lambda_{1}>0$, such that, if $0<\lambda<\lambda_{1}$, then $J$ verifies a local Palais-Smale condition for $c \leq 0$.

Proof. (1) and (2) are immediate. To prove (3), observe that all Palais-Smale sequences for $J$ with $c \leq 0$ must be bounded; then, by Lemma 2.3 , if $\lambda$ verifies $S^{N / p} / N-K \lambda^{\beta} \geq 0$ there exists a convergent subsequence.

Observe that, by (2), if we find some negative critical value for $J$, then we have a negative critical value of $F$.

Now, we will construct an appropriate mini-max sequence of negative critical values for the functional $J$.

Lemma 4.3. Given $n \in \mathbf{N}$, there is $\varepsilon=\varepsilon(n)>0$, such that

$$
\gamma\left(\left\{u \in W_{0}^{1, p}(\Omega): J(u) \leq-\varepsilon\right\}\right) \geq n .
$$

Proof. Fix $n$, let $E_{n}$ be an $n$-dimensional subspace of $W_{0}^{1, p}(\Omega)$. We take $u_{n} \in E_{n}$, with norm $\left\|\nabla u_{n}\right\|_{p}=1$. For $0<\rho<R_{0}$, we have

$$
J\left(\rho u_{n}\right)=F\left(\rho u_{n}\right)=\frac{1}{p} \rho^{p}-\frac{1}{p^{*}} \rho^{p^{*}} \int|u|^{p^{*}}-\frac{\lambda}{\rho} \rho^{q} \int|u|^{q} .
$$

$E_{n}$ is a space of finite dimension; so, all the norms are equivalent. Then, if we define

$$
\begin{aligned}
& \alpha_{n}=\inf \left\{\int|u|^{p^{*}}: u \in E_{n},\left\|\nabla u_{n}\right\|_{p}=1\right\}>0, \\
& \beta_{n}=\inf \left\{\int|u|^{q}: u \in E_{n},\left\|\nabla u_{n}\right\|_{p}=1\right\}>0,
\end{aligned}
$$

we have

$$
J\left(\rho u_{n}\right) \leq \frac{1}{p} \rho^{p}-\frac{\alpha_{n}}{p^{*}} \rho^{p^{*}}-\frac{\lambda \beta_{n}}{q} \rho^{q},
$$

and we can choose $\varepsilon$ (which depends on $n$ ), and $\eta<R_{0}$, such that $J(\eta u) \leq-\varepsilon$ if $u \in E_{n}$, and $\|\nabla u\|_{p}=1$.

Let $S_{\eta}=\left\{u \in W_{0}^{1, p}(\Omega):\|\nabla u\|_{p}=\eta\right\} . S_{\eta} \cap E_{n} \subset\left\{u \in W_{0}^{1, p}(\Omega): J(u) \leq-\varepsilon\right\}$; therefore, by Proposition 4.1,

$$
\gamma\left(\left\{u \in W_{0}^{1, p}(\Omega): J(u) \leq-\varepsilon\right\}\right) \geq \gamma\left(S_{\eta} \cap E_{n}\right)=n .
$$

This lemma allows us to prove the existence of critical points. 
Lemma 4.4. Let $\Sigma_{k}=\left\{C \subset W_{0}^{1, p}(\Omega)-\{0\}, C\right.$ is closed, $\left.C=-C, \gamma(C) \geq k\right\}$. Let $c_{k}=\inf _{C \in \Sigma_{k}} \sup _{u \in C} J(u), K_{c}=\left\{u \in W_{0}^{1, p}(\Omega): J^{\prime}(u)=0, J(u)=c\right\}$, and suppose $0<\lambda<\lambda_{1}$, where $\lambda_{1}$ is the constant of Lemma 4.2.

Then, if $c=c_{k}=c_{k+1}=\cdots=c_{k+r}, \gamma\left(K_{c}\right) \geq r+1$.

(In particular, the $c_{k}$ 's are critical values of $J$. )

Proof. In the proof, we will use Lemma 4.3, and a classical deformation lemma (see $[\mathrm{Be}])$.

For simplicity, we call $J^{-\varepsilon}=\left\{u \in W_{0}^{1, p}(\Omega): J(u) \leq-\varepsilon\right\}$. By Lemma 4.3, $\forall k \in \mathbf{N}, \exists \varepsilon(k)>0$ such that $\gamma\left(J^{-\varepsilon}\right) \geq k$.

Because $J$ is continuous and even, $J^{-\varepsilon} \in \Sigma_{k}$; then, $c_{k} \leq-\varepsilon(k)<0, \forall k$. But $J$ is bounded from below; hence, $c_{k}>-\infty \forall k$.

Let us assume that $c=c_{k}=\cdots=c_{k+r}$. Let us observe that $c<0$; therefore, $J$ verifies the Palais-Smale condition in $K_{c}$, and it is easy to see that $K_{c}$ is a compact set.

If $\gamma\left(K_{c}\right) \leq r$, there exists a closed and symmetric set $U, K_{c} \subset U$, such that $\gamma(U) \leq r$. (We can choose $U \subset J^{0}$, because $c<0$.)

By the deformation lemma, we have an odd homeomorphism

$$
\eta: W_{0}^{1, p}(\Omega) \rightarrow W_{0}^{1, p}(\Omega),
$$

such that $\eta\left(J^{c+\delta}-U\right) \subset J^{c-\delta}$, for some $\delta>0$. (Again, we must choose $0<\delta<-c$, because $J$ verifies the Palais-Smale condition on $J^{0}$, and we need $J^{c+\delta} \subset J^{0}$.) By definition,

$$
c=c_{k+r}=\inf _{C \in \Sigma_{k+r}} \sup _{u \in C} J(u) .
$$

Then, there exists $A \in \Sigma_{k+r}$, such that $\sup _{u \in A} J(u)<c+\delta$; i.e., $A \subset J^{c+\delta}$, and

$$
\eta(A-U) \subset \eta\left(J^{c+\delta}-U\right) \subset J^{c-\delta} .
$$

But $\gamma(\overline{A-U}) \geq \gamma(A)-\gamma(U) \geq k$, and $\gamma(\eta(\overline{A-U})) \geq \gamma(\overline{A-U}) \geq k$.

Then, $\eta(\overline{A-U}) \in \Sigma_{k}$. And this contradicts (4.4); in fact,

$$
\eta(\overline{A-U}) \in \Sigma_{k} \quad \text { implies } \sup _{u \in \eta(\overline{A-U})} J(u) \geq c_{k}=c .
$$

This lemma proves the following result:

Theorem 4.5. Given problem (1.1), with $1<q<p$, there exists $\lambda_{1}>0$, such that, for $0<\lambda<\lambda_{1}$, there exists infinitely many solutions.

Remark 4.6. (1) For the truncated functional $J$, a result of Brezis-Oswald [BO] for $p=2$, which is extended to a general case for Diaz-Saa [D-S], proves the uniqueness of nontrivial positive solutions.

Then, the solutions that we find change the sign, except for those associated with $c_{1}$. In fact, $c_{1}=\inf J(u)$, and, if $c_{1}=J\left(u_{0}\right)$, then $c_{1}=J\left(\left|u_{0}\right|\right)$. That 
is, $\left|u_{0}\right|$ is a nonnegative solution, and, by the maximum principle (see [T]), is strictly positive on $\Omega$.

Observe that there is not a uniqueness result for the nontruncated functional $F$. It remains open the question of the existence of positive solutions with positive energy (solutions as those of $\S 3$, for $p<q<p^{*}$ ).

(2) It is possible to make another proof of Theorem 4.5, if we replace the truncation of $F$ by a special construction of the deformation function $\eta$. In fact, we can take $\eta$ which acts on $B\left(0, R_{0}\right) \subset W_{0}^{1, p}(\Omega)$, and is the identity otherwise; then we must define

$$
\bar{\Sigma}_{k}=\left\{C \subset B\left(0, R_{0}\right)-\{0\}: C \text { closed, symmetric, } \gamma(C) \geq k\right\} .
$$

(3) The critical values that we have obtained are negative, and $F$ verifies the Palais-Smale condition for $c<0$; then, it is easy to see that the set of solutions of Theorem 4.5, is a compact set.

Now, we can show that it is not possible to extend the Palais-Smale condition that we have proved.

Take $x_{0} \in \Omega$, and the balls $B_{j}=B\left(x_{0}, j \delta\right) \subset \Omega$, and the following $C_{0}^{\infty}\left(\mathbf{R}^{N}\right)$ functions:

$$
\begin{array}{ll}
\varphi_{\delta} \equiv 1 \quad \text { on } \Omega-B_{3}, & \xi_{\delta} \equiv 1 \quad \text { on } B_{1}, \\
\varphi_{\delta} \equiv 0 \quad \text { on } B_{2}, & \xi_{\delta} \equiv 0 \text { on } \Omega-B_{2}, \\
\left|\nabla \varphi_{\delta}\right|<2 / \delta, & \left|\nabla \xi_{\delta}\right|<2 / \delta .
\end{array}
$$

We define $\phi_{\delta}=\varphi_{\delta} v+\xi_{\delta} w_{\varepsilon}$, where $F^{\prime}(v)=0$, and $F(v)<0, F\left(w_{\varepsilon}\right) \underset{\varepsilon \rightarrow 0}{\longrightarrow}$ $S^{N / p} / N$ and $F^{\prime}\left(w_{\varepsilon}\right) \underset{\varepsilon \rightarrow 0}{\longrightarrow} 0$. (Take $w_{\varepsilon}=S^{(N-p) / p^{2}} v_{\varepsilon}$, with $v_{\varepsilon}$ defined in $\S 3$.) Later, we shall choose $\varepsilon=\varepsilon(\delta) \underset{\delta \rightarrow 0}{\longrightarrow} 0$.

Then, $F\left(\phi_{\delta}\right)=F\left(\varphi_{\delta} v\right)+F\left(\xi_{\delta} w_{\varepsilon}\right)$, and we can show that $F\left(\phi_{\delta}\right) \underset{\delta \rightarrow 0}{\longrightarrow} C<$ $S^{N / p} / N$, with $F^{\prime}\left(\phi_{\delta}\right) \underset{\delta \rightarrow 0}{\longrightarrow} 0$.

But it is not possible to find a convergent subsequence of $\left\{\phi_{\delta}\right\}$, because $\phi_{\delta}-v$ but

$$
\begin{aligned}
\left\|\phi_{\delta}-v\right\|_{W_{0}^{1, p}(\Omega)} & =\left\|\left(\varphi_{\delta}-1\right) v+\xi_{\delta} w_{\varepsilon}\right\|_{W_{0}^{1, p}(\Omega)} \\
& \geq\left\|\xi_{\delta} w_{\varepsilon}\right\|_{W_{0}^{1, p}(\Omega)}-\left\|\left(\varphi_{\delta}-1\right) v\right\|_{W_{0}^{1, p}(\Omega)}>M>0
\end{aligned}
$$

with $M$ independent of $\delta$.

\section{A PROBLEM WITHOUT SYMMETRY}

We shall consider the following model problem:

$$
\begin{aligned}
-\operatorname{div}\left(|\nabla u|^{p-2} \nabla u\right) & =\lambda|u|^{q-2} u+f(x), \quad \lambda>0, \\
\left.u\right|_{\delta \Omega} & =0,
\end{aligned}
$$

where $\Omega$ is a rectangle in $\mathbf{R}^{N}$, and $p<q<p^{*}, 1<p<N$. When $f \equiv 0$, there are infinitely many solutions $\forall \lambda>0$. In the proof, we use a mini-max type theory, as in $\S 4$, because the associated functional is even. 
When $f \not \equiv 0$, the associated functional is

$$
I(u)=\frac{1}{p} \int_{\Omega}|\nabla u|^{p}-\frac{\lambda}{q} \int_{\Omega}|u|^{q}-\int_{\Omega} f u .
$$

We cannot apply the previous method, because $I$ is not even; however, it is possible to make use of the method developed by P. Rabinowitz in the case $p=2$ (see [R1 and R2]). For the sake of completeness, we will include here the proofs of the more interesting steps.

The point is the lack of control on the nonsymmetric part of the functional $I$; that is, $I(u)-I(-u)$. The idea is to find some appropriated truncation of $I$, in order to obtain a functional $J$, in which the nonsymmetric part can be estimated, such that the existence of critical points for $J$ implies the existence of critical points for $I$. We start with an a priori estimate, which gives us the idea to make the truncation.

Lemma 5.1. There exists a constant $A=A\left(\|f\|_{p^{\prime}}\right)>0$ such that, if $I^{\prime}(u)=0$, then

$$
\frac{\lambda}{q} \int_{\Omega}|u|^{q} \leq A\left(|I(u)|^{p}+1\right)^{1 / p} .
$$

(The proof is an easy adaptation of those made in [R1].) With this estimate, we make the following truncation: Let $\chi: \mathbf{R} \rightarrow[0,1]$ such that

$$
\begin{aligned}
& \chi(x)=0, \quad x \geq 2, \\
& \chi(x)=1, \quad x \leq 1, \\
& -2 \leq \chi^{\prime}(x) \leq 0
\end{aligned}
$$

and

$$
\psi(u)=\chi\left\{\frac{(\lambda / q) \int|u|^{q}}{2 A\left(|I(u)|^{p}+1\right)^{1 / p}}\right\} .
$$

Define

$$
J(u)=\frac{1}{p} \int_{\Omega}|\nabla u|^{p}-\frac{\lambda}{q} \int_{\Omega}|u|^{q}-\int_{\Omega} \psi(u) f u .
$$

In particular, Lemma 5.1 implies that, if $I^{\prime}(u)=0$, then $J^{\prime}(u)=0$. However, we need just the converse. The main properties of $J$ are the following (for the proof, see $[R 1])$ :

\section{Lemma 5.2.}

(i) $J \in \mathbf{C}^{1}\left(W_{0}^{1, p}(\Omega), \mathbf{R}\right)$.

(ii) $\exists \beta>0, \beta=\beta\left(\|f\|_{p^{\prime}}\right)$, such that $|J(u)-J(-u)| \leq \beta\left(|J(u)|^{1 / q}+1\right)$.

(iii) $\exists M_{0}>0$, such that if $J(u) \geq M_{0}$, and $J^{\prime}(u)=0$, then $\psi(v) \equiv 1$ in a neighbourhood of $u$ (that is, $J(u)=I(u)$, and $\left.J^{\prime}(u)=I^{\prime}(u)=0\right)$.

(iv) $\exists M_{1} \geq M_{0}$ such that $J$ verifies a local Palais-Smale condition for $C>M_{1}$. That is, if we have a sequence $\left\{u_{k}\right\} \subset W_{0}^{1, p}(\Omega)$ such that $J\left(u_{k}\right) \rightarrow C$ and $J^{\prime}\left(u_{k}\right) \rightarrow 0$, then there exists a convergent subsequence $\left\{u_{k_{j}}\right\} \subset\left\{u_{k}\right\}$. 
According to (iii), if we find some critical value for $J$, and it is large enough, then we have a solution of problem (5.1). We will prove a stronger result: we construct a sequence of critical values for $J$, which tends to infinity.

To simplify the notation, we assume $\Omega=(0,1)^{N}$. Let $E_{k}$ be the $k$ dimensional subspace of $W_{0}^{1, p}(\Omega)$, generated by the first $k$ functions of the basis

$$
\left\{\left(\sin k_{1} \pi x_{1} \cdots \sin k_{N} \pi x_{N}\right), k_{i} \in \mathbf{N}, i=1, \ldots, N\right\}
$$

(see [G-P.2]).

In this finite dimensional subspace, it is easy to prove that it is possible to construct an increasing sequence of numbers $R_{j}>0$ (as big as we wish), such that

$$
J(u) \leq 0 \quad \text { if } u \in E_{j} \cap B_{R_{j}}^{C} .
$$

Let $D_{j}=B_{R_{j}} \cap E_{j}$, and define

$$
\begin{gathered}
G_{j}=\left\{h \in \mathbf{C}\left(D_{j}, W_{0}^{1, p}(\Omega)\right): h \text { is odd },\left.h\right|_{\delta B_{R_{j}} \cap E_{j}}=\mathrm{Id}\right\}, \\
b_{j}=\inf _{h \in G_{j}} \max _{u \in D_{j}} J(h(u)) .
\end{gathered}
$$

First, we prove that the sequence $\left\{b_{j}\right\}$ is well defined, and increasing:

Proposition 5.3. Let $b_{k}$ defined by (5.8). Then, there exists a constant $\beta>0$, such that

$$
b_{k} \geq \beta k^{\gamma}
$$

where $\gamma=p q / N(q-p)-1$.

Proof. Given $h \in G_{k}$, and $\rho<R_{k}$, we can prove that $h\left(D_{k}\right) \cap \delta B_{\rho} \cap E_{k-1}^{C} \neq \varnothing$. In fact, it suffices to show that $\gamma\left(h\left(D_{k}\right) \cap \delta B_{\rho}\right) \geq k$, and apply property (8) of Proposition 4.1. Let $A=\left\{x \in D_{k}: h(x) \in B_{\rho}\right\}$. It is clear that $0 \in A$, because $h$ is odd; then, we define $A_{0}$ the component of $A$ containing $0 . A_{0}$ is a bounded and symmetric neighbourhood of 0 in $E_{k}$; then, $\gamma\left(\delta A_{0}\right)=k$.

Moreover, $h\left(\delta A_{0}\right) \subset \delta B_{\rho}$. If not, given $x \in \delta A_{0}$ such that $h(x) \in B_{\rho}$, if $x \in D_{k}$, there exists a neighbourhood of $x, U$, such that $h(U) \subset B_{\rho}$. Then, $x \notin \delta A_{0}$. Hence, $x \in \delta D_{k}$; but $\left.h\right|_{\delta D_{k}}=$ Id, and this implies that $\|h(x)\|=\|x\|=R_{k}>\rho$, a contradiction.

Now, if we define $B=\left\{x \in D_{k}: h(x) \in B_{\rho}\right\}$, we have $\delta A_{0} \subset B$, and

$$
\gamma\left(h\left(D_{k}\right) \cap \delta B_{\rho}\right)=\gamma(h(B)) \geq \gamma(B) \geq \gamma\left(\delta A_{0}\right)=k .
$$

Note that the condition " $h$ is even" is essential to obtain this result; then, it is an important ingredient in the definition of $G_{k}$.

Let $u \in \delta B_{\rho} \cap E_{k-1}^{C}$; then

$$
J(u) \geq \frac{1}{p} \int_{\Omega}|\nabla u|^{p}-\frac{\lambda}{q} \int_{\Omega}|u|^{q}-C_{1}\|u\|_{p}
$$


where $C_{1}=C_{1}\left(\|f\|_{p^{\prime}}\right)$. By using the Gagliardo-Nirenberg inequality,

$$
\left(\int_{\Omega}|u|^{q}\right)^{1 / q} \leq C\left(\int_{\Omega}|\nabla u|^{p}\right)^{a / p}\left(\int_{\Omega}|u|^{p}\right)^{(1-a) / p}
$$

with $a=(N / p)(1-p / q)$, we get

$$
J(u) \geq \frac{1}{p} \int_{\Omega}|\nabla u|^{p}-C_{1}\left(\int_{\Omega}|\nabla u|^{p}\right)^{q a / p}\left(\int_{\Omega}|u|^{p}\right)^{q(1-a) / p}-C_{2}\left(\int_{\Omega}|u|^{p}\right)^{1 / p} .
$$

Moreover, $u \in E_{k-1}^{C}$; hence,

$$
\|u\|_{p} \leq C\|\nabla u\|_{p} / k^{1 / N}
$$

(see [G-P.2] for the proof). Finally, by (5.11) and (5.12), we obtain

$$
\begin{aligned}
J(u) & \geq \frac{1}{p} \rho^{p}-C_{1}\left(\frac{C}{k^{q(1-a) / N}}\right) \rho^{q}-\left(\frac{C_{3}}{k^{1 / N}}\right) \rho \\
& =\rho^{p}\left(\frac{1}{p}-\frac{C_{2}}{k^{q(1-a) / N}} \rho^{q-p}\right)-\frac{C_{3}}{k^{1 / N}} \rho .
\end{aligned}
$$

Now, we choose

$$
\rho_{k}=\left\{\frac{k^{q(1-a) / N}}{2 p C_{2}}\right\}^{1 /(q-p)} ;
$$

therefore,

$$
J(u) \geq \frac{1}{2 p} \rho_{k}^{p}-\frac{C_{3}}{k^{1 / N}} \rho_{k} \geq C k^{p q(1-a) / N(q-p)}
$$

for $k$ large enough. Then, by equations (5.13) and (5.10), we get $\forall h \in G_{k}$, $\max _{u \in D_{k}} J(h(u)) \geq C k^{\gamma}$, where

$$
\gamma=\frac{p q(1-a)}{N(q-p)}=\frac{p q}{N(q-p)}-1 .
$$

And this implies (5.9).

If we try to prove that $b_{k}$ is a critical value for $J$, we find an important obstruction, unless $f \equiv 0$. In fact, in the case $f \not \equiv 0$, the associated functional $J$ is not even, and the deformation lemma give us a homeomorphism $\eta$ which is not odd. Then, given $h \in G_{k}$, in general $\eta \circ h \notin G_{k}$, and the classical proof does not work.

However, the sequence $\left\{b_{k}\right\}$ allows us to prove that other mini-max sequences that we will construct are well defined and verifies the appropriate estimates. Define

$$
\begin{aligned}
& U_{k}=\left\{u=t e_{k+1}+w, t \in\left[0, R_{k+1}\right], w \in B_{R_{k+1}} \cap E_{k},\|u\| \leq R_{k+1}\right\}, \\
& \Lambda_{k}=\left\{H \in C\left(U_{k}, W_{0}^{1, p}(\Omega)\right),\left.H\right|_{D_{k}} \in G_{k},\right. \\
& \left.\left.H\right|_{\left(\delta B_{R_{k+1}} \cap E_{k+1}\right) \cup\left(B_{R_{k+1}} \cap B_{R_{k}}^{C} \cap E_{k}\right)}=\mathrm{Id}\right\},
\end{aligned}
$$




$$
c_{k}=\inf _{H \in \Lambda_{k}} \max _{u \in U_{k}} J(H(u)) .
$$

These mini-max values have the same problem as the $b_{k}$ 's: if $\left.H\right|_{D_{k}} \in G_{k}$, then $\left.H\right|_{D_{k}}$ is odd, but $\left.\eta \circ H\right|_{D_{k}}$ is not odd, in general. However, it is clear that $c_{k} \geq b_{k}$ (compare (5.16) and (5.8)); and if $c_{k}>b_{k}$, we can solve our problem, as the following proposition shows:

Proposition 5.4. If $c_{k}>b_{k}>M_{1}$ (where $M_{1}$ is the constant of Lemma 5.2 (iv)), given $\delta \in\left(0, c_{k}-b_{k}\right)$, we define

$$
\begin{gathered}
\Lambda_{k}(\delta)=\left\{H \in \Lambda_{k} \text { such that } J(H(u)) \leq b_{k}+\delta, \forall u \in D_{k}\right\}, \\
c_{k}(\delta)=\inf _{H \in \Lambda_{k}(\delta)} \max _{u \in U_{k}} J(H(u)) .
\end{gathered}
$$

Then, $c_{k}(\delta)$ is a critical value for $J$.

Proof. By definition (5.8) it is clear that $\Lambda_{k}(\delta) \neq \varnothing$. And, by (5.16) and (5.18), it is also clear that $c_{k}(\delta) \geq c_{k}$. Suppose that $c_{k}(\delta)$ is not a critical value and take $\varepsilon<\frac{1}{2}\left(c_{k}-b_{k}-\delta\right)$.

By the classical deformation theorem, we obtain the homeomorphism $\eta: W_{0}^{1, p}(\Omega) \rightarrow W_{0}^{1, p}(\Omega)$, with the following properties:

$$
\begin{gathered}
\eta\left(J^{c_{k}(\delta)+\varepsilon}\right) \subset J^{c_{k}(\delta)-\varepsilon}, \\
\eta(u)=u, \quad \text { if } u \notin J^{-1}\left(\left[c_{k}(\delta)-2 \varepsilon, c_{k}(\delta)+2 \varepsilon\right]\right) .
\end{gathered}
$$

Note that if $u \in D_{k}$ and $H \in \Lambda_{k}(\delta)$, then

$$
J(H(u)) \leq b_{k}+\delta<c_{k}-2 \varepsilon,
$$

that is, if $H \in \Lambda_{k}(\delta)$, by (5.20) and (5.21), then $\left.\eta \circ H\right|_{D_{k}}=\left.H\right|_{D_{k}} \in G_{k}$. Then, we have solved the problem of the lack of symmetry in $\eta$. Now, it is easy to conclude: we prove that $\eta \circ H \in \Lambda_{k}(\delta)$, and find a contradiction between (5.18) and (5.19).

Finally, it remains to prove that it is impossible to have $c_{k}=b_{k}, \forall k$.

Proposition 5.5. If $c_{k}=b_{k}, \forall k \geq k^{*}$, there exist some constants $C>0$, and $k^{\prime} \geq k^{*}$, such that

$$
b_{k} \leq C k^{q /(q-1)}, \quad \forall k \geq k^{\prime} .
$$

Proof. Basically, the idea is to use that $D_{k+1}=\left(U_{k}\right) \cup\left(-U_{k}\right)$, and if $H \in \Lambda_{k}$, it is possible to extend it to a function of $G_{k}$.

By (5.15) and (5.16), we can choose $H \in \Lambda_{k}$ such that

$$
\max _{u \in U_{k}} J(H(u)) \leq c_{k}+\varepsilon=b_{k}+\varepsilon,
$$

and, by (5.8), taking the extension of $H$, we have

$$
b_{k+1} \leq \max _{u \in D_{k+1}} J(H(u)) .
$$


If the maximum is attained at $U_{k}$, then

$$
b_{k+1} \leq \max _{u \in D_{k+1}} J(H(u))=\max _{u \in U_{k}} J(H(u)) \leq c_{k}+\varepsilon \leq b_{k}+\varepsilon .
$$

If the maximum is attained on $-U_{k}$, we can use estimate (iii) of Lemma 5.2, in the following way: Suppose $\max _{u \in D_{k+1}} J(H(u))=J(H(w))$, for some $w \in-U_{k}$. Then,

$$
\begin{aligned}
J(H(-w)) & \geq J(H(w))-\beta\left(|J(H(-w))|^{1 / q}+1\right) \\
& \geq b_{k+1}-\beta\left(\left(b_{k}+\varepsilon\right)^{1 / q}+1\right) \geq b_{k+1}-\beta\left(\left(b_{k+1}+\varepsilon\right)^{1 / q}+1\right)>0,
\end{aligned}
$$

for $k$ large. And, if $J(H(-w))>0$,

$$
\begin{aligned}
b_{k+1} & \leq J(H(w))=J(-H(-w)) \\
& \leq J(H(-w))+\beta\left(J(H(-w))^{1 / q}+1\right) \\
& \leq\left(b_{k}+\varepsilon\right)+\beta\left(\left(b_{k}+\varepsilon\right)^{1 / q}+1\right) .
\end{aligned}
$$

Getting $\varepsilon \rightarrow 0$ at (5.24) and (5.25), we obtain $b_{k+1} \leq b_{k}+\beta\left(b_{k}^{1 / q}+1\right)$. Finally, this inequality implies (5.22); the proof can be made by induction.

Proposition 5.5, together with Proposition 5.3 and Proposition 5.4, prove the following theorem:

Theorem 5.6. Problem (5.1), when $q /(q-1)<p q / N(q-p)-1$, has infinitely many solutions, which correspond to a sequence of critical values of the functional (5.2), the sequence tending to infinity.

Remark 5.7. Note that, for $p=2$, Theorem 5.6 is contained in Theorem 10.4 in [R2].

Acknowledgments. The authors thank the referee for his valuable advice to revise the manuscript, and for his suggestions concerning the inclusion of some references [G-V, A-P-S, E, and O].

\section{REFERENCES}

[A] A. Anane, Simplicité et isolation de la première valeur propre du p-laplacien avec poids., C. R. Acad. Sci. Paris Sér. I Math. 305 (1987), 725-728.

[A-E] J. P. Aubin and I. Ekeland, Applied nonlinear analysis, Wiley, New York, 1984.

[A-P-S] F. V. Atkinson, L. A. Pelletier, and J. Serrin, unpublished work.

[B] H. Brézis, Some variational problems with lack of compactness, Proc. Berkeley Sympos. Nonlinear Functional Analysis, 1985.

[Ba] G. Barles, Remarks on the uniqueness results of the first eigenvalue of the p-Laplacian, Ann. de Toulouse (to appear).

[Be] V. Benci, On critical point theory for indefinite functionals in the presence of symmetries, Trans. Amer. Math. Soc. 274 (1982), 533-572.

[B-F] V. Benci and D. Fortunato, Bifurcation from the essential spectrum for odd variational operators, Confer. Sem. Mat. Univ. Bari 178 (1981).

[Bha] T. Bhattacharya, Radial symmetry of the first eigenfunction for the p-laplacian in the ball, Preprint. 
[B-N] H. Brézis and L. Nirenberg, Positive solutions of nonlinear elliptic equations involving critical Sobolev exponents, Comm. Pure Appl. Math. 36 (1983), 437-477.

[B-O] H. Brézis and L. Oswald, Remarks on sublinear elliptic equations, Inst. Math. Appl. Preprint Ser. 112 (1984).

[DiB] E. Di Benedetto, $C^{1, \alpha}$ local regularity of weak solutions of degenerate elliptic equations, Nonlinear Anal. 7 (1983), 827-850.

[D-S] I. Diaz and J. E. Saa, Uniqueness of nonnegative solutions for elliptic nonlinear diffusion equations with a general perturbation term, Proc. VII CEDYA, Santander, 1985.

[E] H. Egnell, Existence and nonexistence results for m-laplace equations involving critical Sobolev exponents, Arch. Rational Mech. Anal. 104 (1988), 57-77.

[G-P.1] J. Garcia Azorero and I. Peral Alonso, Existence and non-uniqueness for the p-laplacian: Nonlinear eigenvalues, Comm. Partial Differential Equations 12 (1987), 1389-1430.

[G-P.2] _ Comportement asymptotique des valeurs propres du p-laplacien, C. R. Acad. Sci. Paris Sér. I Math. 307 (1988), 75-78.

[G-V] M. Guedda and L. Veron, Quasilinear elliptic equations involving critical Sobolev exponents (to appear).

[L1] P. L. Lions, The concentration-compactness principle in the calculus of variations. The limit case, part 1, Rev. Mat. Iberoamericana 1 (1985), 145-201.

[L2] _ The concentration-compactness principle in the calculus of variations. The limit case, part 2, Rev. Mat. Iberoamericana 1 (1985), 45-121.

[O] M. Otani, Existence and nonexistence of nontrivial solutions of some nonlinear degenerate equations, J. Funct. Anal. 76 (1988), 140-159.

[P-S] P. Pucci and J. Serrin, A general variational identity, Indiana Univ. Math. J. 35 (1986), 681-703.

[R1] P. H. Rabinowitz, Multiple critical points of perturbed symmetric functionals, Trans. Amer. Math. Soc. 272 (1982).

[R2] _ Minimax methods in critical point theory with applications to differential equations, CBMS Regional Conf. Ser. in Math., no. 65, Amer. Math. Soc., Providence, R.I., 1986.

[T] P. Tolksdorff, Regularity for a more general class of quasilinear elliptic equations, J. Differential Equations 51 (1984), 126-150.

Departamento de Matemáticas, Universidad Autónoma de Madrid, 28049 Madrid, SPAIN 